\title{
DESIGNING AND IMPLEMENTATION OF SIMPLE RETINAL VESSEL SEPARATION BASED ON ADAPTIVE LOCAL THRESHOLDING
}

\author{
Mohammad Sami ${ }^{1}$, Dr. Avinash Gour ${ }^{2}$ \\ ${ }^{1}$ Research Scholar, Dept. of Electronics and Communication Engineering, Sri Satya Sai University \\ of Technology \& Medical Sciences, Sehore, Bhopal-Indore Road, Madhya Pradesh, India. \\ ${ }^{2}$ Research Guide, Dept. of Electronics and Communication Engineering, Sri Satya Sai University of \\ Technology \& Medical Sciences, Sehore, Bhopal Indore Road, Madhya Pradesh, India \\ Email: samisbit@gmail.com
}

Keywords:

\section{Retinal,}

Threshold technique,

Post processing,

Preprocessing,

Proposed method.

\begin{abstract}
A BS T RA C T
The retina has one of the items like retinal blood vessels. The attributes of the retinal vessels have their unique size and shape. Illnesses endured by people, with cardiovascular disorders, hypertension and diabetic retinopathy can be recognized by looking at these blood vessels. A computerized framework that can recognize blood vessels from different items in the retina is here introduced. It comprises of segmentation, preprocessing and exactness computations.
\end{abstract}

Citation: Mohammad Sami, Dr. Avinash Gour (2020). Design and Implementation Of Simple Retinal Vessel Separation Based On Adaptive Local Thresholding. International Journal of Advanced Multidisciplinary Scientific Research (IJAMSR) ISSN:2581-4281, 2(8), August 2019, pp 9 - 19 
IJAMSR 2 (8) www.ijamsr.com CrossRef: https://doi.org/10.31426/ijamsr.2019.2.8.1812

\section{Introduction}

Retinal have a key impact in helpful imaging. Segmentation is used as a piece of the numerous applications, for instance, examination of physical structure, medical screening during an assessment of convolution, stenosis and angiogenesis. In clinical examination, segmentation helps the patients to distinguish the degree of the seriousness of the diseases ${ }^{[1]}$. In any case, the aforementioned applications request a satisfactory segmentation technique that can confine assorted sizes of the vessels just as perceive inconsistencies in the vessels for better appraisal. A bit of an available techniques are manual based [2]. Manual segregation of the vessel and non-vessel pixels is infuriating, complex and tedious, especially during the examination of colossal and composite databases when diverged from mechanized/programmed segmentation.

Disregarding the way that the automated techniques are pondered to be exact and snappy, regardless of all that they defy challenges, for instance, inconvenience in perceiving vessels from the non-vessels as a result of obstruction made by blockage tissues, inconvenience in sectioning assorted widths of vessels especially undesirable vessels in view of presence of ancient rarities in medical images, which prompts misclassification. The vascular system of retina photo contains the huge subtleties which are used for the distinguishing proof and investigation of various retinal disorders, for instance, hypertension, glaucoma and diabetes [3]. The eye's master used fundus camera for catching retinal photo of the patients. These retinal photos are utilized by the ophthalmologist for reviews, screening and investigation of different retinal disorders. The segmentation of blood vessels in retina images show huge vascular varieties which are utilized for acknowledgment and conclusions of different ophthalmic abnormalities. The structure of vessel and non-vessel pixels is exceptionally homogenous in retinal images, which make vessels difficult to disengage from the foundation pixels. Therefore, it is mandatory to use a suitable picture segmentation structure for exact extraction of retinal vasculature. These methodologies rely upon the picture structures, for instance, the cross-sectional profiles, indistinguishable force areas and limits ${ }^{[4] .}$ 
IJAMSR 2 (8) www.ijamsr.com CrossRef: https://doi.org/10.31426/ijamsr.2019.2.8.1812

Typical retina contains neuroretina and retinal shade epithelium. The neuroretina comprises of external and inward fragments of photoreceptors (poles and cones), outside restricting film, external atomic layer, external plexiform layer, inward atomic layer, inward plexiform layer, ganglion cell layer, nerve fiber layer and inner constraining layer ${ }^{[5,6]}$. There is an expansive range of retinal pathology including inborn abnormalities, dystrophies, degenerations (remarkably age-related macular degeneration), retinal vascular infections, poison levels, incendiary ailments, neoplasms, retinal separation, injury and retinal contribution of foundational sicknesses. This section presents a couple of major neurotic procedures in retinal illnesses, particularly forms that are amiable to pharmacotherapeutics ${ }^{[7]}$.

The worldwide binarization technique is normally quicker than the neighbourhood versatile strategy and the worldwide strategy will give great outcomes for explicit climate conditions, for example, object recognition in winter condition. In these cases ${ }^{[8-10]}$, obtained images have uniform differentiation conveyance of frontal area and foundation and little variety in light. We are searching for a compelling technique for object identification in infrared images in testing conditions, for example, summer conditions or in a urban situation, where there is a deficiency of objects of intrigue. In these cases, we utilized nearby mean procedures and neighbourhood change methods. The investigation results are introduced with the goal that we can all the more likely pick which strategy ought to be utilized or what blend of these past methods to utilize $^{[11][12] .}$

\section{Extraction of Blood Vessels in Retinal} Images Using Four Different Techniques: -

a) Pre-processing

- Experiment 1

- Experiment 2

b) Post-processing

\section{Proposed Method}

This presented novel method for retinal blood vessels extraction, involves 3 stages: prepreparing, segmenting and post-handling. With the end goal of vessel extraction, the green channel of RGB [13-15] picture experience various stages. The green channel is picked in light of the fact that red and blue channel experiences helpless illuminance. Here, every phase are answerable for extraction of blood vessel are clarified below. 
International Journal of Advanced Multidisciplinary Scientific Research (IJAMSR) ISSN:2581-4281 Volume 2, Issue 8, August, 2019

IJAMSR 2 (8) $\quad$ www.ijamsr.com CrossRef: https://doi.org/10.31426/ijamsr.2019.2.8.1812

\section{International Journal of \\ Advanced Multidisciplinary Scientific Research (IJAMSR) ISSN:2581-4281}

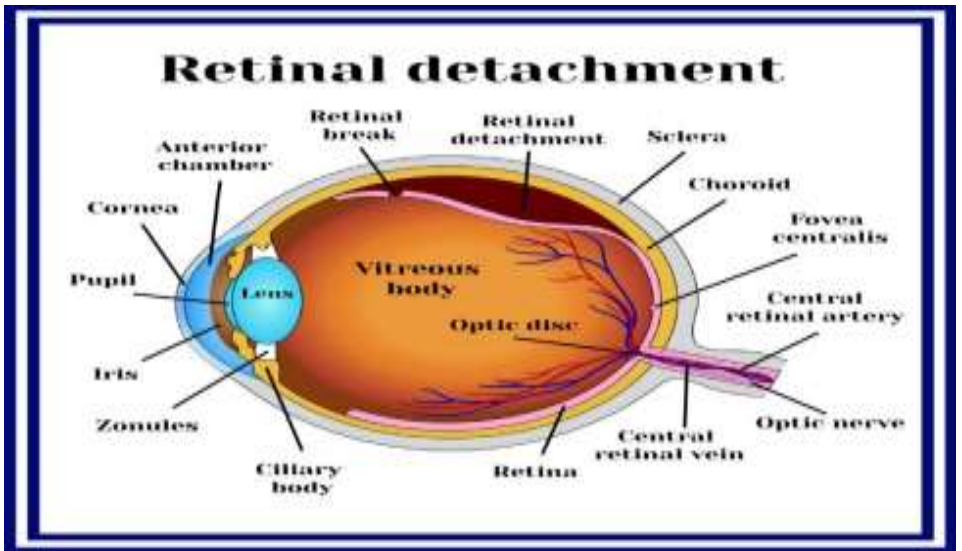

Figure 1. Retinal image

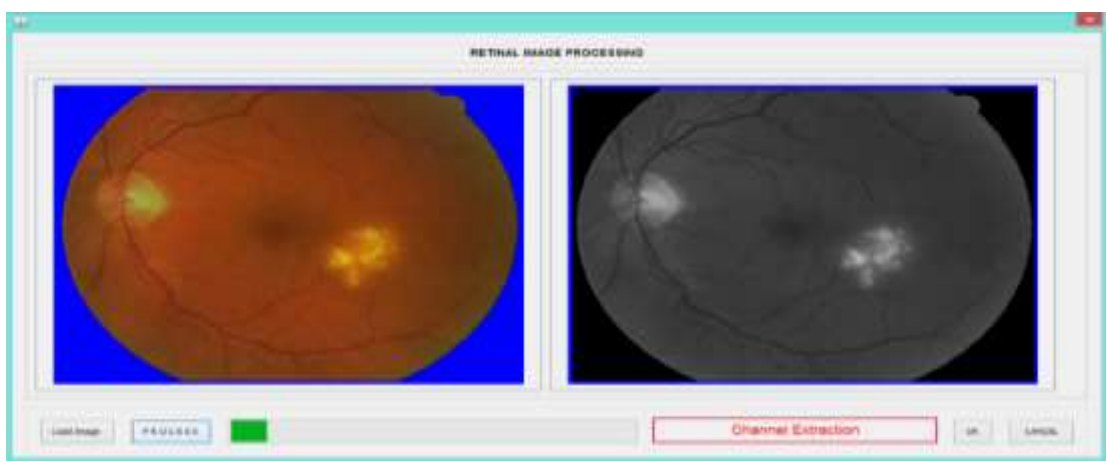

Figure 2. Green channel image extraction

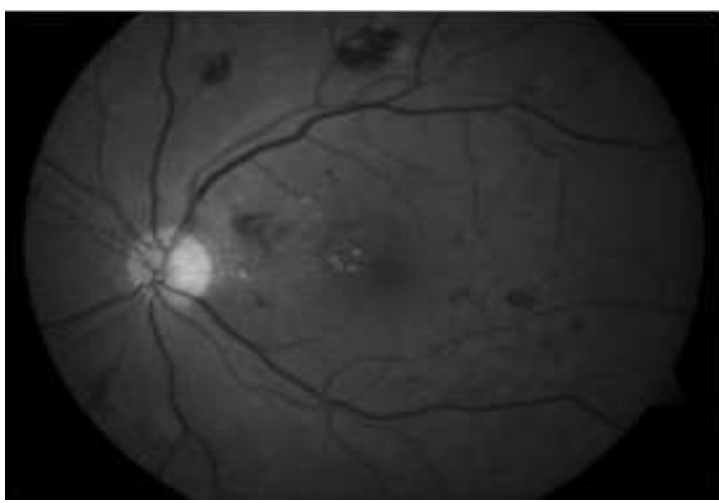

(a)

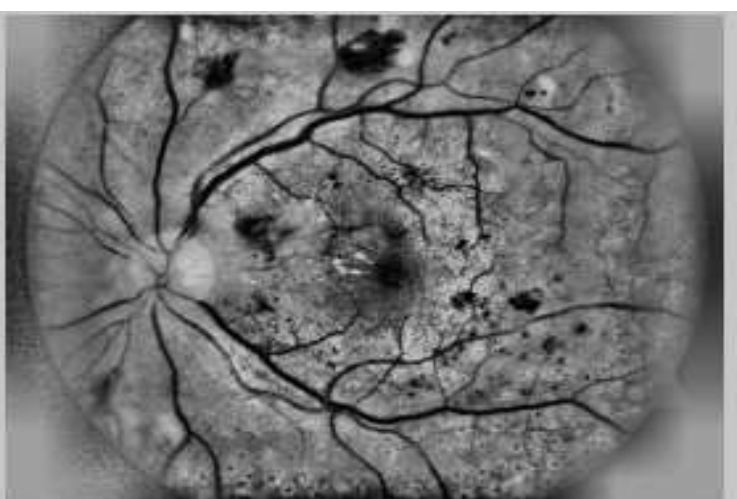

(b)

Figure 3. (a) Image of adaptive histogram equalization

(b)Image of Median filtered Method of Adaptive Local Thresholdin 
IJAMSR 2 (8) www.ijamsr.com CrossRef: https://doi.org/10.31426/ijamsr.2019.2.8.1812

This technique is based upon a simple concept. A parameter called the brightness threshold is chosen and applied to the image a $[\mathrm{m}, \mathrm{n}]$ as follows:

\section{If $a[m, n] \geq \theta \quad a[m, n]=$ object $=1$ \\ Else $\quad a[m, n]=$ background $=0$}

This version of the algorithm assumes that we are interested in light objects on a dark background. For dark objects on a light background we would use:

\section{If $a[m, n]<\theta \quad a[m, n]=$ object $=1$ \\ Else $\quad a[m, n]=$ background $\quad=0$}

The output is the label "object" or "background" which, due to its dichotomous nature, can be represented as a Boolean variable "1" or "0". In principle, the test condition could be based upon some other property than simple brightness (for example, If (Redness $\{\mathrm{a}[\mathrm{m}, \mathrm{n}]\}>=\theta$ red), but the concept is clear.

The central question in thresholding then becomes: how do we choose the threshold $\theta$ ? While there is no universal procedure for threshold selection that is guaranteed to work on all images, there are a variety of alternatives.
Fixed Threshold - One alternative is to use a threshold that is chosen independently of the image data. If it is known that one is dealing with very high-contrast images where the objects are very dark and the background is homogeneous and very light, then a constant threshold of 128 on a scale of 0 to 255 might be sufficiently accurate. By accuracy we mean that the number of falsely-classified pixels should be kept to a minimum.

Histogram-Derived Thresholds -In most cases the threshold is chosen from the brightness histogram of the region or image that we wish to segment. An image and its associated brightness histogram are shown in Figure 2.

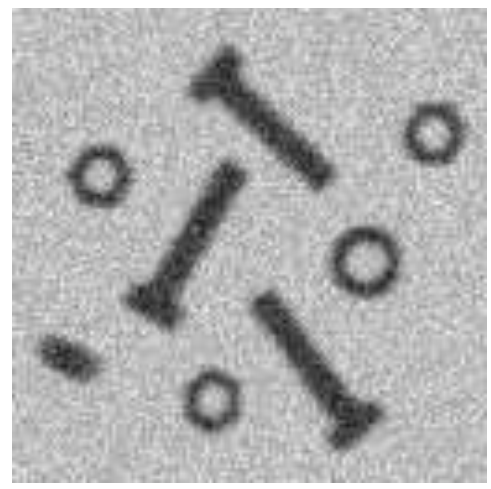

(a) Image to be threshold 
IJAMSR 2 (8) www.ijamsr.com CrossRef: https://doi.org/10.31426/ijamsr.2019.2.8.1812

\section{$12 \quad$ International Journal of \\ I J A M S R \\ Advanced Multidisciplinary Scientific Research (IJAMSR) ISSN:2581-4281}

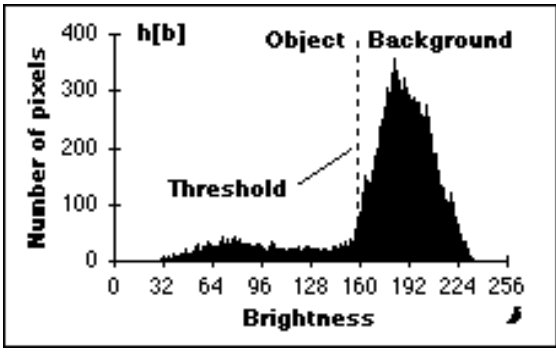

(b) Brightness histogram of the image

Figure 4: Pixels below the threshold (a [m, n] $<\theta)$ will be labeled as object pixels; those above the threshold will be labeled as background pixels.

A variety of techniques have been devised to automatically choose a threshold starting from the gray-value histogram, $\{h[b] \mid b=0,1,2 B$ 1\}. Some of the most common ones are presented below. Many of these algorithms can benefit from a smoothing of the raw histogram data to remove small fluctuations but the smoothing algorithm must not shift the peak positions. This translates into a zero-phase smoothing algorithm given below where typical values for $\mathrm{W}$ are 3 or 5 :

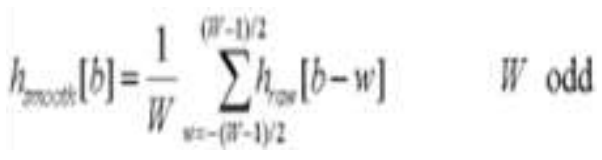

Isodata algorithm: This iterative technique for choosing a threshold was developed by Ridler and Calvard. The histogram is initially segmented into two parts using a starting threshold value such as $\theta 0=2 \mathrm{~B}-1$, half the maximum dynamic range. The sample mean (mf, 0) of the gray values associated with the foreground pixels and the sample mean $(\mathrm{mb}, 0)$ of the gray values associated with the background pixels are computed. A new threshold value $\theta 1$ is now computed as the average of these two sample means. The process is repeated, based upon the new threshold, until the threshold value does not change any more. In formula:

$\theta_{k}=\left(m_{j, k-1}+m_{i, k-1}\right) / 2$ until $\theta_{k}=\theta_{k-1}$

\section{Background-symmetry algorithm- This} technique assumes a distinct and dominant peak for the background that is symmetric about its maximum. The technique can benefit from smoothing as described in eq. . . . The maximum peak (maxp) is found by searching for the maximum value in the histogram. The algorithm then searches on the non-object pixel side of that maximum to find a $\mathrm{p} \%$ point as in eq..

In Figure $2 b$, where the object pixels are located to the left of the background peak at brightness 183 , this means searching to the right of that peak to locate, as an example, the $95 \%$ value. At this brightness value, $5 \%$ of the pixels lie to the right (are above) that value. 
IJAMSR 2 (8) www.ijamsr.com CrossRef: https://doi.org/10.31426/ijamsr.2019.2.8.1812

This occurs at brightness 216 in Figure $2 b$. Because of the assumed symmetry, we use as a threshold a displacement to the left of the maximum that is equal to the displacement to the right where the $\mathrm{p} \%$ is found. For Figure $2 b$ this means a threshold value given by 183 $(216-183)=150$. In formula:

This technique can be adapted easily to the case where we have light objects on a dark, dominant background. Further, it can be used if the object peak dominates and we have reason to assume that the brightness distribution around the object peak is symmetric. An additional variation on this symmetry theme is to use an estimate of the sample standard deviation ( $\mathrm{s}$ in eq. (37)) based on one side of the dominant peak and then use a threshold based on $\theta=\operatorname{maxp}+/-1.96 \mathrm{~s}$ (at the $5 \%$ level) or $\theta=\operatorname{maxp}+/-2.57 \mathrm{~s}$ (at the $1 \%$ level). The choice of "+" or "-" depends on which direction from maxp is being defined as the object/background threshold. Should the distributions be approximately Gaussian around maxp, then the values 1.96 and 2.57 will, in fact, correspond to the 5\% and $1 \%$ level.

Triangle algorithm- This technique due to Zack is illustrated in Figure 3. A line is constructed between the maximum of the histogram at brightness $b_{\max }$ and the lowest value $b_{\min }=(p=0) \%$ in the image. The distance $\mathbf{d}$ between the line and the histogram $h[b]$ is computed for all values of $b$ from $b$ $=b_{\min }$ to $\mathrm{b}=\mathrm{b}_{\max }$. The brightness value $\mathrm{Bo}$ where the distance between $\mathrm{h}[\mathrm{Bo}]$ and the line is maximal is the threshold value, that is, $\theta=$ Bo. This technique is particularly effective when the object pixels produce a weak peak in the histogram[16][17].

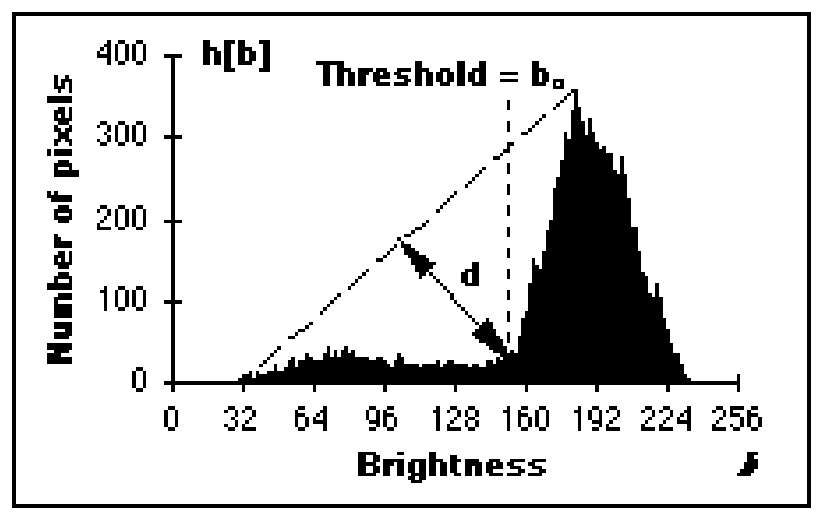

Figure 5: The triangle algorithm is based on finding the value of $b$ that gives the maximum distance $\mathbf{d}$.

The three procedures described above give the values $\theta=139$ for the Isodata algorithm, $\theta=150$ for the background symmetry algorithm at the $5 \%$ level, and $\theta=152$ for the triangle algorithm for the image.

Thresholding does not have to be applied to entire images but can be used on a region by 
IJAMSR 2 (8) www.ijamsr.com CrossRef: https://doi.org/10.31426/ijamsr.2019.2.8.1812

region basis. Chow and Kaneko developed a variation in which the $\mathrm{MxN}$ image is divided into non-overlapping regions. In each region a threshold is calculated and the resulting threshold values are put together (interpolated) to form a thresholding surface for the entire image. The regions should be of "reasonable" size so that there are a sufficient number of pixels in each region to make an estimate of the histogram and the threshold. The utility of this procedure, like so many others, depends on the application at hand. Thresholding is viewed as a kind of the most notable, basic and exact method to image segmenting when all is said in done and to medical image segmentation specifically. Though the items course of action in the common part figures aspects generally undistinguishable, the game plan of articles remembering the tissues and organs for the medical images are typically more noticeable. Along these lines, thresholding segmentation strategies are utilized broadly in the exploration which includes the medical picture segmentation, where various organs and tissues are denoted in various dim stages. Regularly, thresholding strategies, in their essential structure, look for a worldwide worth (stage) that ideally expands the detachment between various classes (various tissues for our situation) in the picture. The adequacy of thresholding with a worldwide level shows if the articles in the image viable have very much characterized regions and if the dark levels can be congregated around values with least impedance.

Lopsided brightening, sub-par nature of camera, source material, curios/twists, and anatomical items with multi-classes and half and half highlights make the worldwide thresholding for the whole retinal image a significant hotspot for segmentation blunders. In addition, since retinal image shows delicate progress between various dim levels, lopsided brightening or commotion bends, the central segmentation mistakes start to show up because of pixel-wise methodology that embraced by worldwide thresholding, in particular, the pixels that have same dark levels (pixel force) will be divided into a similar anatomical item, which is viewed as a longstanding issue of worldwide thresholding with a solitary hard worth.

To determine these difficulties, district savvy thresholding methods have been recommended for instance of retinal vessels recognizable proof, created and executed by means of various procedures which can be ordered into three significant classes: measurable, 
IJAMSR 2 (8) www.ijamsr.com CrossRef: https://doi.org/10.31426/ijamsr.2019.2.8.1812

\section{International Journal of \\ I J A M S R \\ Advanced Multidisciplinary Scientific Research (IJAMSR) ISSN:2581-4281}

information based and fluffy based versatile thresholding.

\section{Results}

The current retinal blood vessel segmentation methodologies are ordered, and depicted in the last area. We examined different procedures utilized in these method for blood vessels in retinal image segmentation and analyze execution aftereffect of the methodologies. These methods were assessed utilizing freely accessible datasets. Different segmentation methodologies of retinal vessels follow comparable techniques: every methodology starts by pre-processing step, where the green layer (or dark) is separated from the crude shading retinal image, and afterward the complexity of the image is upgraded. Working steps signifies the core of calculation, where the various procedures ordered in last area are utilized. At last, in the post-processing stages, the underlying portioned image experiences steps of smoothing and edge preserving and improvement.

With respect to segmentation classes is either a best method of calculation to confront all presentation measurements in high segmenting accomplishment, nor a best scientific plan to do as such. Choosing whether the method is best contingent upon a lot of elements including,

a) Attained accuracy

b) Time and computational complexity

c) Robustness
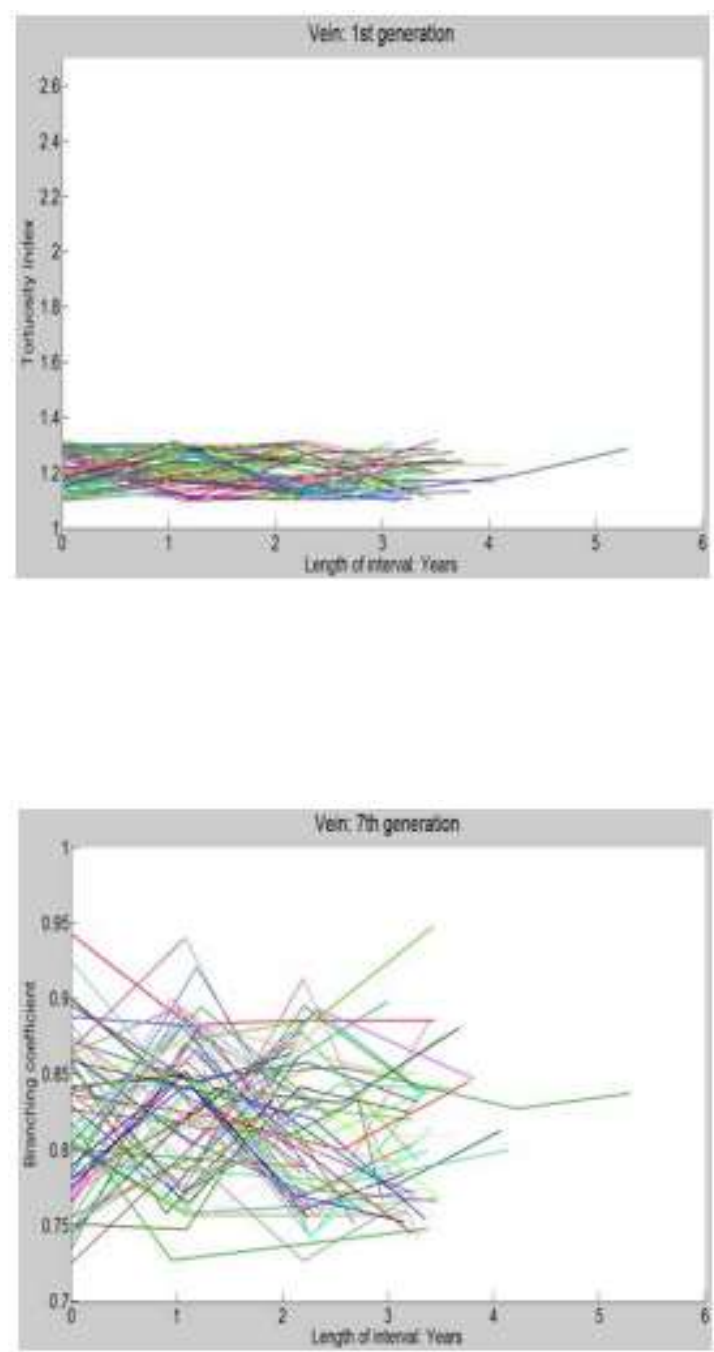

Figure 6: Comparison result of existing vs. proposed vessel segmentation techniques 
IJAMSR 2 (8) www.ijamsr.com CrossRef: https://doi.org/10.31426/ijamsr.2019.2.8.1812

Here, some images are randomly selected from the medical databases where tested and trained for producing the better result of segmentation. As per the above result the process time also faster while comparing with other existing techniques like clustering, the exact recognition and segmentation of the retinal vascular structure shapes the foundation of an assortment of computerized PC supported frameworks for screening and conclusion of ophthalmologic and cardiovascular illnesses. Despite the fact that lot of promising methods has been created and executed, there is still space for investigation development in blood vessel segment methods, particularly for boisterous and neurotic retinal images outside the set number of retinal images accessible in open data items. All things considered, applications, retinal vessels segmentation frameworks won't supplant the specialists' job in conclusion; rather, they will upgrade the determination precision and lessen the outstanding task at hand of the ophthalmologists. In this manner, huge size of patients' pictures can be prepared with high conclusion precision with equivalent time.

\section{Conclusion}

The extraction of the retinal blood vessels has been a vivaciously examined zone in present age. The ideal confinement of the retinal vasculature builds up the establishment of various mechanized PC supported frameworks for examination and location of ophthalmologic and cardiovascular disorders. In spite of the fact that lot of promising methods and procedures have been created, there is still chance to show signs of improvement in blood vessel extraction draws near.

\section{References}

1) A. Imran, J. Li, Y. Pei, J. J. Yang, and Q. Wang, "Comparative analysis of vessel segmentation techniques in retinal images, "IEEE Access, vol. 7, pp. 114862 114887, 2019.

2) Kolb, H. Simple Anatomy of the Retina. In Web vision: The Organization of the Retina and Visual System; Kolb, H., Fernandez, E., Nelson, R., Eds.; University of Utah Health Sciences Center: Salt Lake City, UT, USA, 1995; Available online: http://europepmc.org/books/NBK11533;jse ssionid $=4 C 8 B A D 63 F 75 E A D 49 C 21 B C 65 E$ 2AE5F6F3 (accessed on 22 January 2018).

3) H. A. Leopold, J. Orchard, J. S. Zelek, and V. Lakshminarayanan, "PixelBNN: augmenting the PixelCNN with batch normalization and the presentation of $a$ fast architecture for retinal vessel segmentation," Journal of Imaging, vol. 5, no. 2, p. 26, 2019. 
IJAMSR 2 (8) www.ijamsr.com CrossRef: https://doi.org/10.31426/ijamsr.2019.2.8.1812

4) MESSIDOR: Methods for Evaluating Segmentation and Indexing Techniques Dedicated to Retinal Ophthalmology, 2004. Available

online: http://www.adcis.net/en/DownloadThird-Party/Messidor.html (accessed on 22 January 2018).

5) T. Chakraborti, D. K. Jha, A. $S$. Chowdhury, and X. Jiang, "Aself-adaptive matched filter for retinal blood vessel detection," Machine Vision and Applications PP 2014.

6) Kolb, H. Simple Anatomy of the Retina, 2012.

Available

online: http://webvision.med.utah.edu/book /part-i-foundations/simple-anatomy-of-theretina/ (accessed on 22 January 2018).

7) Soomro TA, Gao J, Khan T, Hani AFM, Khan MAU, Paul M. Computerized approaches for the detection of diabetic retinopathy using retinal fundus images: a survey. Pattern Analysis and Applications. 2017.

8) M.M. Fraz, A. Basit, S.A. Barman

Application of morphological bit planes in retinal blood vessel extraction J Digit Imaging, 26 (2) (2013), pp. 274-286.

9) Jusoh F, Haron H, Ibrahim R, Azemin M (2016) An overview of retinal blood vessels segmentation. Advanced computer and communication engineering technology. Springer, Berlin.

10) Kolb, H. Simple Anatomy of the Retina. In Web vision: The Organization of the Retina and Visual System; Kolb, H., Fernandez, E., Nelson, R., Eds.; University of Utah Health Sciences Center: Salt Lake City, UT, USA, 1995; Available online: http://europepmc.org/books/NBK11 533;jsessionid $=4 C 8 B A D 63 F 75 E A D 49 C 21$ BC65E2AE5F6F3 (accessed on 22 January 2018).

11) Akram, M.U., Khan, S.A.: Multilayered thresholding-based blood vessel segmentation for screening of diabetic retinopathy. Eng. Computer. 29, 165-173 (2013)
12) Liskowski, P., Krawiec, K.: Segmenting retinal blood vessels with deep neural networks. IEEE Trans. Med. Imaging 35(11), 2369-2380 (2016)

13). Fraz MM, Remagnino $P$, Hoppe A, Uyyanonvara B, Rudnicka AR, et al. (2012) Blood vessel segmentation methodologies in retinal images-a survey. Computer Methods Programs Biomed 108(1): 407433.

14) Alberola, J.M.; del Val, E.; Costa, A.; Novais, P.; Julian, V. A genetic algorithm for group formation in elderly communities. AI Commun. 2018, 31, 409425.

15) Chamoso, P.; De Paz, J.F.; De La Prieta, F.; Bajo Pérez, J. Agreement technologies applied to transmission towers maintenance. AI Commun. 2017, 30, 8398.

16) E. Sorantin, C. Halmai, B. Erdohelyi, K. Palagyi, L. Nyul, K. Olle, B. Geiger, F. Lindbichler, G. Friedrich, and K. Kiesler, "Spiral-ct-based assessment of tracheal stenoses using 3-dskeletonization", IEEE Trans. on Med. Img., vol. 21, pp. 263-273, March 2002.

17) Fraz MM, Remagnino $P$, Hoppe A, Uyyanonvara B, Rudnicka AR, et al. (2012) Blood vessel segmentation methodologies in retinal images-a survey. Comput Methods Programs Biomed 108(1): 407433. 